\title{
UV laser induced ferroelectric domain inversion in lithium niobate single crystals
}

\author{
S. Mailis \\ Optoelectronics Research Centre, University of Southampton, Highfield, SO17 1BJ, \\ U.K. \\ E-mail: sm@orc.soton.ac.uk
}

\begin{abstract}
UV radiation in the spectral region beyond $\sim 320 \mathrm{~nm}$ is strongly absorbed by lithium niobate single crystals. The strong absorption and the consequent localized heating of the crystal triggers physical processes which can affect the state of the material not only by changing the refractive index, as shown in the past, but also by changing its ferroelectric disposition. UV laser irradiation of the polar surfaces in particulars can induce or inhibit the inversion of ferroelectric domains. A summary of these UV light induced effects and their utility in the micro-structuring of this very important optical ferroelectric crystal will be presented here.
\end{abstract}

Keywords: Lithium niobate, ferroelectric domains, direct laser writing, micro-structuring

PACS: 77.84.Ek, 77.22.Ej, 81.16.-c

\section{Introduction}

Since it's synthesis lithium niobate $(\mathrm{LN})$ has been in the forefront of photonics world. It is a ferroelectric crystal which remains stable for a wide range of temperatures (Curie temperature $\sim 1150^{\circ} \mathrm{C}$ ) and possesses substantially large nonlinear and electro-optic coefficients [1]. It is therefore an important technological material which is used in a wide range of applications involving optical switching [2], nonlinear optical frequency generation [3], surface acoustic wave (SAW) filtering [4] and many others. For this reason it is commercially available today in large quantities and in excellent and most importantly consistent quality.

$\mathrm{LN}$ has been the subject of extensive research for the last 30 years and there is a large amount of research publications to prove it. Initially the bulk of research was directed towards the standardization of growth in order to achieve reproducibly good quality crystals. Later, the effort was directed towards the development of photonic devices such as optical modulators and frequency converters. For this reason numerous methods for the fabrication of optical waveguides were identified such as in-diffusion of titanium metal $[5,6]$, proton exchange $[7,8]$, ion implantation [9] and ultra-fast laser direct writing [10] to name a few.

Regarding optical non-linear applications, such as frequency conversion, in $\mathrm{LN}$ is possible to use the birefringence of the crystal in order to achieve phase matching of the nonlinear process. However, birefringence phase matching does not permit the usage of the highest nonlinear coefficient $\left(\mathrm{d}_{33}\right)$. Ferroelectric domain engineering provided a remedy to this problem by offering the possibility for quasi-phase-matching [11] (QPM) in periodically poled LN (PPLN) crystals. Ferroelectric domain engineering has been developed primarily for nonlinear frequency conversion by quasi-phase-matching [3] however other applications are beginning to emerge such as the performance improvement of electro-optic [12] and acousto-optic devices [13, 14]. Differential etching of domain engineered crystals offers also the capability for surface micro-structuring $[15,16]$.

The conventional method for ferroelectric domain inversion in LN is by applying an external electric filed E-field) of sufficient amplitude to exceed the coercive field. Spatially selective 
domain inversion is obtained by modulating the amplitude of the E-field either by directly structuring the electrodes which are used for the application of the electric field or by introducing dielectric layers, i.e. photoresist, between a uniform planar electrode and the crystal surface. Recently however, it was shown that it is possible to modify locally the effective coercive field by irradiating the crystal with a laser beam while applying an external electric filed $[17,18]$. This is due to a photo-induced space charge field which adds to the externally applied E-field [19, 20]. Light assisted poling (LAP) leads to spatially selective domain inversion in a direct writing fashion.

\subsection{UV laser induced effects}

The absorption edge in congruently melt, undoped LN crystals is located around $350 \mathrm{~nm}$. At a wavelength range between $305 \mathrm{~nm}-244 \mathrm{~nm}$ the light is absorbed within a layer of few microns, or even less. The strong absorption of the UV light is resulting in a rapid local heating of the crystal accompanied often by surface damage, even meting at laser intensities in the range of $\sim 300-400 \mathrm{~kW} / \mathrm{cm}^{2}$. The local laser induced heating at temperatures up to the melting point is due to the relatively poor heat conductivity of LN crystals. As a result steep temperature gradients are formed during the UV laser irradiation.

The diffusion of heat after UV laser irradiation and the formation of temperature gradients has been studied in [21] in an attempt to explain the observation that optical waveguides could be directly written in LN by UV laser irradiation at $244 \mathrm{~nm}$ [22]. The literature value for the absorption depth at $244 \mathrm{~nm}$ radiation is of order $\sim 30 \mathrm{~nm}$. The short absorption depth suggests that the optical waveguide formation cannot be explained by a direct optical photorefractive effect as the volume where a refractive index change occurs in order to support a waveguide mode should be much larger that the volume where the light is absorbed. However, the model which was presented in [21] suggests that the heated volume, due to the UV laser irradiation, is much larger as compared to the UV radiation absorption depth. This prediction offers the alternative route of a heat driven mechanism that could be responsible for the observed refractive index change.

Apart for the refractive index changes UV direct irradiation of the polar surfaces of the crystal induces a range of effects which are relevant to the ferroelectric domain state of the crystal and which originate from the rapid heating cooling cycle which is a consequence of the strong absorption of light in the crystal. The impact of UV radiation on the poling behaviour of LN and more specifically the UV laser induced domain inversion and the UV laser-induced inhibition of poling will be discussed in this paper. Furthermore the utility of these UV laser enabled processes in the fabrication of micron and sub-micron scale micro-structures in LN will be demonstrated.

\section{2. .All optical poling}

Differential etching between the opposite polar faces of $\mathrm{LN}(-\mathrm{z},+\mathrm{z})$ in hydrofluoric (HF) acid is a standard method for the visualization of inverted ferroelectric domain distributions in LN where normally the $-\mathrm{z}$ face etches at a specific (temperature dependent) rate while the $+\mathrm{z}$ remains practically unaffected by the acid [23] thus creating a surface relief pattern that corresponds to the ferroelectric domain distribution. Early experiments investigating the interaction of UV laser radiation with the surface of LN crystals showed that the spatially selective exposure of the -z polar face of the crystal with c.w. or pulsed UV laser could change the chemical etching behaviour of this particular face in $\operatorname{HF}[24,25]$. More specifically UV irradiation was observed to completely inhibit the etching of the -z crystal face hinting that local domain inversion could have been taken place.

The c.w. UV laser irradiation experiments were performed in a scanning fashion where the surface of the crystal sample was scanned in front of the focussed laser beam. In this way it was possible to vary the exposure time, by adjusting the scanning speed and the intensity by adjusting the laser power and the laser beam focussing characteristics. In the pulsed laser case the irradiation was often performed through an absorption or phase mask. 


\subsection{All optical poling with c.w. laser irradiation}

All optical poling using c.w. laser radiation $(244 \mathrm{~nm})$ has been observed following scanned or static exposure of the $-\mathrm{z}$ face of $\mathrm{LN}$ [26]. In these experiments the UV laser beam from a frequency doubled argon-ion laser was focussed to a spot diameter of $\sim 1.5 \mu \mathrm{m}$ on the surface of the crystal while the laser powers used was within the narrow range of $25-30 \mathrm{~mW}$ which is below the damage threshold. At higher laser powers the surface becomes severely damaged while at lower powers no effect is observed.

Following UV irradiation the crystal was etched in HF acid revealing a surface relief pattern which corresponded to the laser irradiated pattern. Figure 1 shows scanning electro microscopy (SEM) images of the surface relief pattern of the laser exposed $-\mathrm{z}$ face which is revealed after HF etching. The set of ridges which are shown in figure 1 correspond to the UV laser exposed tracks. It is obvious that the quality of the surface is not the same in all the tracks. Notably the tracks which correspond to lower power appear to be grainy in nature consisting of submicron features while at higher laser powers the corresponding etch-resistant surface appears solid. This is an indication that at lower powers an assembly of inverted nanodomains is formed within the laser irradiated area. At higher powers the nano-domains merge to form a solid inverted domain layer however, surface cracks appears which can be clearly seen in figure $1 \mathrm{a}$, probably as a result of the expansion of the heated volume with respect to the surrounding crystal volume that remains at lower temperature.

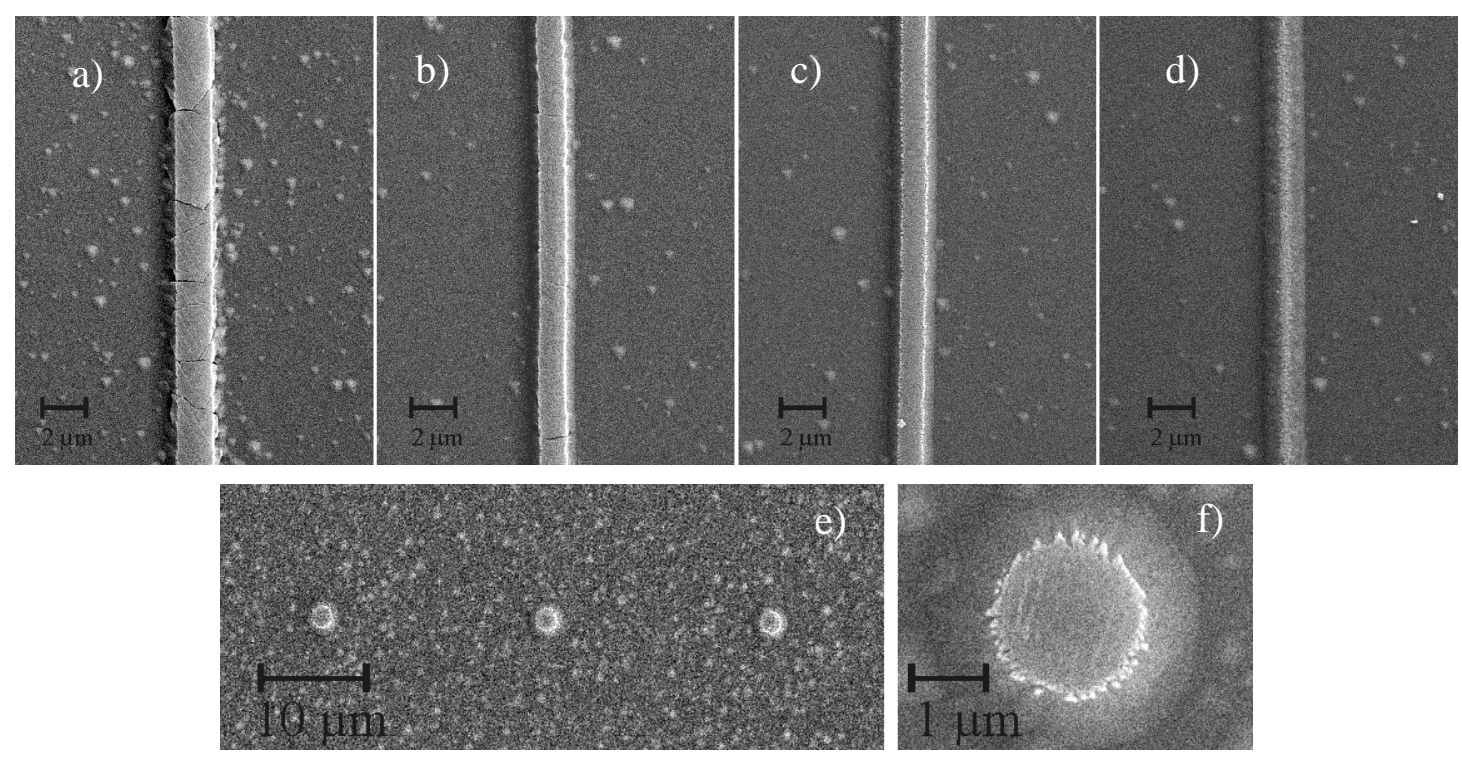

Figure 1. a-d SEM images of direct UV laser poled tracks corresponding to different laser powers (with track "a" corresponding to the highest power) as revealed by etching in HF acid. e) Set of isolated laser-poled domains formed by static UV laser exposure, f) higher magnification detail.

In the SEM images of figure1 it can be observed that the writing laser power level has also an impact on the density of the nano-domains. This difference in nano-domain density is more evident in the track shown in image (d) which corresponds to the lowest power. The concentration of the nano-domains is lower here allowing the acid to attack them from the side thus reducing the width of the ridge. Static exposures also yielded isolated inverted domains with a circular profile as shown in the SEM image of figure 2. Direct UV laser domain inversion was also observed in Fe: and Ti: doped crystals.

The role of the laser intensity was seen to be more important than the exposure time, which could be adjusted by the scanning speed. This is an indication that the occurrence of direct poling depends strongly on the peak temperature which is achieved during laser irradiation and not on the duration that the crystal stays at an elevated temperature. This observation points towards a dynamic process which occurs during the heating - cooling cycle of the 
affected crystal volume. It has been proposed that the effect originates from a synergy of electric fields, formed by displaced charge carriers and the pyro-electric field, which adds up to produce a net poling field during the cooling stage of the rapid heating-cooling cycle as a result of the laser irradiation. However, the exact physical processes which take place at such high temperatures as well as the exact values of the electrical conductivity, thermal diffusivity and optical absorption are not accurately known. This reduces the capability of modelling in detail the actual mechanism which is responsible for this effect.
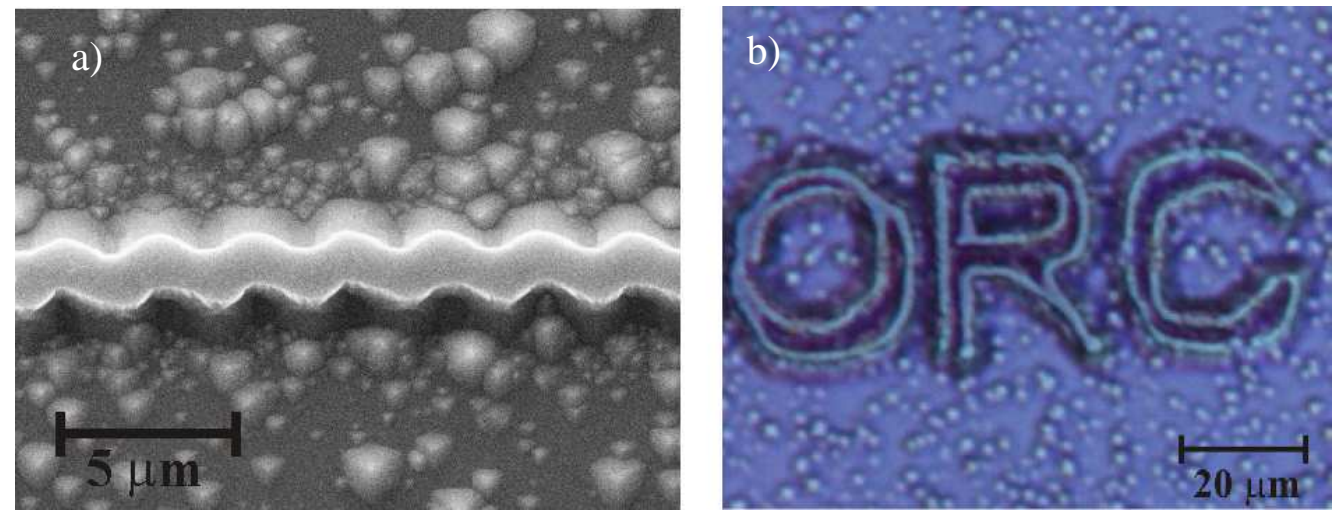

Figure 2. a) SEM image of a UV laser inverted structure with curved edges, b) Optical microscope image of a direct UV poled patter of the letters "ORC" illustrating the flexibility of the method.

The direct write nature of laser poling enhances the flexibility and applicability of this domain engineering method. Furthermore direct UV laser poling of LN does not seem to become restricted by the crystal symmetry as it is often the case in conventional electric field poling. This allows the inverted domain inscription of arbitrary shapes including curvatures. The SEM image shown in figure $2 \mathrm{a}$ exhibits curvatures that are impossible to achieve by conventional electric field domain inversion processes while the optical microscopy image shown in figure $2 \mathrm{~b}$ shows the directly poled letters "ORC" demonstrating the capability for free style domain inscription.

\subsection{All optical poling with pulsed laser irradiation}

A modification of the etch rate of the $-\mathrm{z}$ polar face have been also observed after irradiation with a pulsed UV laser $(248 \mathrm{~nm})$ source with different pulse durations (20 ns and $500 \mathrm{fs}$ ). Again it was observed that for a narrow range of exposure conditions an etch-resistant region could be produced on the surface [27]. Figure 3 shows an SEM image of the irradiated area after brief etching in HF. The exposure in this particular experiment was performed through a metallic hexagonal mesh with hexagonal openings through which the UV light could propagate freely and reach the surface. The metallic mesh was attached to the surface of the sample. Due to contact imperfections however diffraction of the laser radiation occurred at the metal edges resulting in an inhomogeneous exposure of the surface. The impact of the inhomogeneous irradiation on the etch-resistant pattern is clearly observed in the SEM images of figure 3. In all cases the energy fluence of the laser was kept at values below the threshold for ablation.

It is worth noticing that the etch-resistant areas which were obtained in the pulsed laser experiments were always consisting of submicron features which never seemed to be able to merge into a solid surface at least within the range of the experimental conditions that were used. The etching behaviour of these features in HF is consistent with direct ferroelectric domain inversion. Again the strong absorption of intense UV light can raise the temperature locally thus producing the appropriate temperature gradients and the transient electric fields which are capable of poling a surface layer of the crystal. There is however a distinct difference when compared with the c.w. case namely the inability for these etch resistant features to merge and form a continuous, uniform etch resistant area. 
The number of pulses or the repetition rate was not observed to improve dramatically the quality of the direct poling and in some cases additional pulses were seen to have a detrimental effect on the domain inversion.

Direct poling is believed to happen during the cooling stage of the heating/cooling cycle which is induced by the absorbed UV laser radiation. When the full effect is achieved, after the first pulse (or the first few pulses) then any subsequent pulse will be irradiating an already domain inverted surface and might even result in partial back-switching [28]. Additionally, the pulsed lasers which were used in these experiments could only operate at repetition rates up to $100 \mathrm{~Hz}$. According to [21] the duration of the full heat/cooling cycle can be much faster than the $10 \mathrm{~ms}$, which is the minimum time interval between subsequent pulses, hence no cumulative heat effects were expected. This prediction is in accordance with the experimental observation.
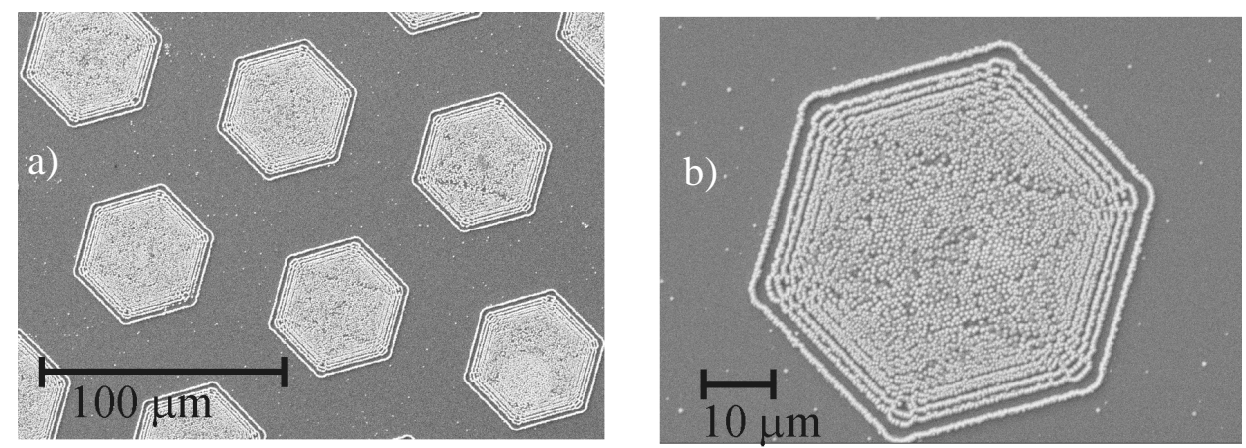

Figure 3. a) a lattice of etch resistant features obtained by pulsed UV laser exposure, b) higher magnification detail of a single hexagon showing the assembly of individual submicron features.

Direct UV laser poling allows the fabrication of inverted domain distributions in systems where the application of an external electric filed is difficult i.e. in thin films and of course maintain the simplicity and flexibility of a direct writing method. In the c.w. case the depth of the inverted domains could be sufficient to overlap with optical waveguide modes. However, even very shallow domains can act as etch stop layers for the fabrication of surface micro/nano-structures in this very important ferroelectric crystal.

\section{UV laser-induced inhibition of poling}

In the previous section it was described how c.w. UV laser $(244 \mathrm{~nm})$ irradiation of the $-\mathrm{z}$ face can achieve direct ferroelectric inversion UV irradiation on the $+z$ polar face of the crystal cannot induce direct domain inversion apart form a very superficial layer with a depth of $\sim 30-50 \mathrm{~nm}$ [26]. UV irradiation of the $+\mathrm{z}$ face however has been observed to inhibit the ferroelectric domain inversion, upon subsequent electric field poling, within the range of the UV laser heated volume as mentioned in the previous sections. Consequently the spatially selective irradiation of the $+\mathrm{z}$ polar face of the crystal produces a multi-domain region, after uniform electric field poling, where the previously irradiated areas of the surface maintains their original polarity [29].

Inhibition of poling was observed after irradiation within a wide spectral range spanning from $305 \mathrm{~nm}$ to $244 \mathrm{~nm}$, always in the strong absorption range, and was observed in both undoped and $\mathrm{MgO}$ doped congruently melting LN crystals. Figure 4 show the HF etched surface of the crystal that have undergone scanned (a) and static (b) exposures, followed by uniform electric field poling. The set of ridges which are shown in figure 4 a correspond to pole inhibited linear domains which formed along UV irradiated tracks while the isolated round features shown in Figure $4 \mathrm{~b}$ correspond to isolated pole inhibited domains as a result of static exposure. The polarity of the different parts of the surface can be deduced by the etching behaviour. The etch-resistant features (ridges and dots) correspond to $\mathrm{a}+\mathrm{z}$ face (which is the original polarity of the surface) while the surrounding area which etches away in HF corresponds to $-\mathrm{z}$ face (which was domain inverted after the electric field poling step). 

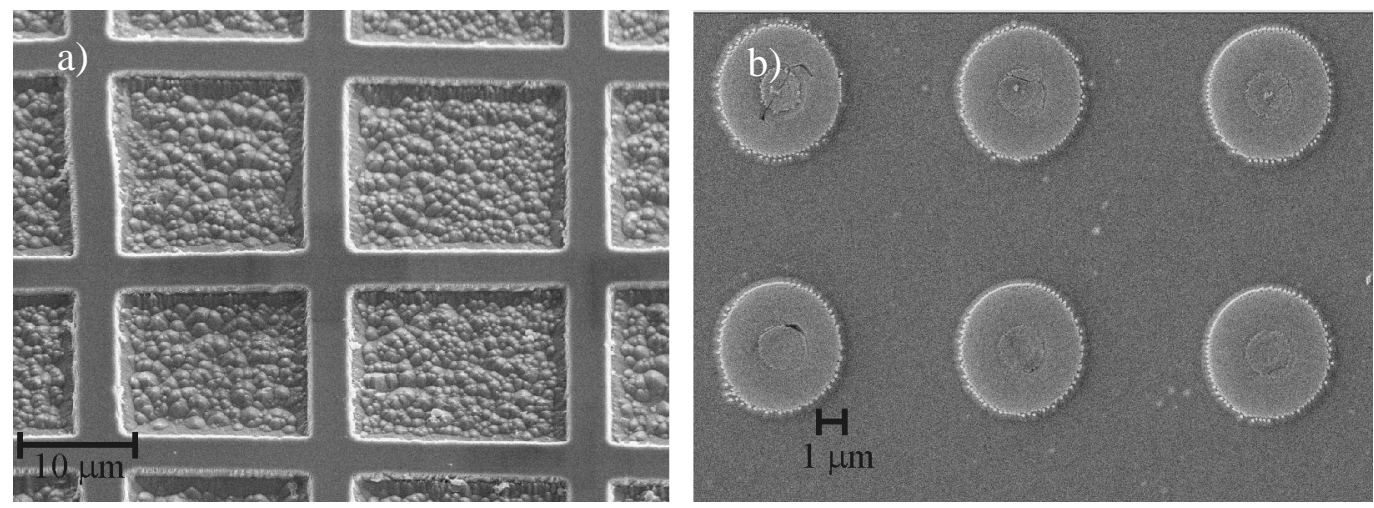

Figure 4. SEM images of a) set of pole inhibited crossed ridges corresponding to UV irradiated tracks, b) isolated pole-inhibited domains corresponding to static exposures of the surface. The surface has been briefly etched in HF.

Detailed investigation of UV irradiated and poled crystals showed that the depth of the pole inhibited domains is of order $\sim$ a few $\mu \mathrm{m}$ [30] and depends upon the UV irradiation conditions (intensity, duration). Their depth is consistent with the range of the laser-heated volume of the crystal. A depth profile of a pole inhibited domain can be seen in the SEM image which is shown in figure 5 . The sample which is shown in figure 5 carries linear poleinhibited domains, corresponding to scanned UV tracks. In order to enhance the depth profile information the sample was wedge-polished at an angle of $\sim 5^{\circ}$ across the pole-inhibited tracks before etched briefly in HF acid to reveal the ferroelectric domain topography. The area where the slope change occurs is indicated by the dash line which is overlaid to the SEM image

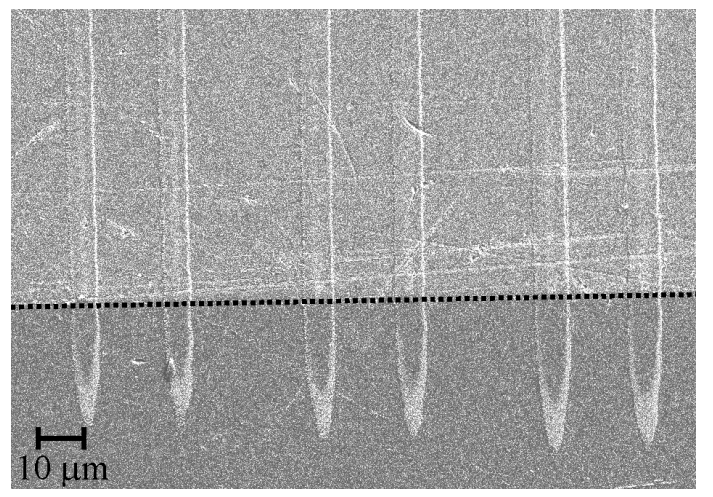

Figure 5. SEM image of the depth profile of pole-inhibited domains as revealed by brief etching in HF. The cross section view was enhanced by wedge polishing at an angle of $\sim 5^{\circ}$. The dash line indicates the position where the slope changes.

Three pairs of tracks are shown in figure 5 with each pair corresponding to different laser intensity. The laser spot size and scanning speed used in this experiment were the same suggesting that the depth of the pole inhibited domains increases with increasing UV laser power. The depths of the pole inhibited domain tracks which are shown in figure 5 range from $2.2 \mu \mathrm{m}$ to $2.7 \mu \mathrm{m}$. This range of depths could overlap well with optical waveguide modes which make this method for domain engineering suitable for waveguide nonlinear quasiphase-matched applications. Typical values of the UV laser power used for the irradiation experiments that resulted in inhibition of poling were within the range of $\sim 30-50 \mathrm{~mW}$ and the spot size of the focussed laser beam was of order $\sim 2 \mu \mathrm{m}$. The ability for producing fine (sub micron) pole-inhibited domains was also investigated by exposing the surface with an interference intensity pattern instead of a plain focussed beam. Brief etching of the surface, after the electric field poling process, revealed a surface relief grating corresponding to the irradiating interference pattern indicating the formation of periodic domains with submicron widths. 
LN exhibits strong absorption in the range of laser wavelengths ( $305 \mathrm{~nm}-244 \mathrm{~nm}$ ) that have been used to demonstrate the effect of poling inhibition. For $244 \mathrm{~nm}$ the absorption depth was estimated to be just $\sim 30 \mathrm{~nm}[31,32]$ which is much shorter than the range of the pole inhibited domains $(\sim 2-3 \mu \mathrm{m})$ excluding a direct photonic mechanism being responsible for the effect. However, the range of the poling inhibited domains is consistent with the temperature spatial distributions resulting by the absorbed UV light [21]. The produced temperature gradients can power the diffusion of mobile species, such as lithium, the diffusion of which has already been proposed to explain the formation of optical waveguides as a result of UV irradiation. It is possible therefore that the redistribution of lithium due to local laser heating plays an important role in the physical mechanism behind poling inhibition. This hypothesis is the subject of current research. Additionally the prospect of superimposing a refractive index change with a surface micro-structure is very attractive for the fabrication of ridge waveguide photonic circuits.

Bulk ferroelectric domain inversion has been used in past for fabricating surface microstructures due to the dependence of the etch rate (in HF) on the sign of the polar surface. Additional differential etching processes along the three identical (due to the three fold symmetry) y-directions allow further micro-structuring capability [33]. However, even inverted ferroelectric domains of limited depth such as the ones achieved by direct laser poling and poling inhibition can provide the means for surface micro-structuring as they constitute an etch stop layer.
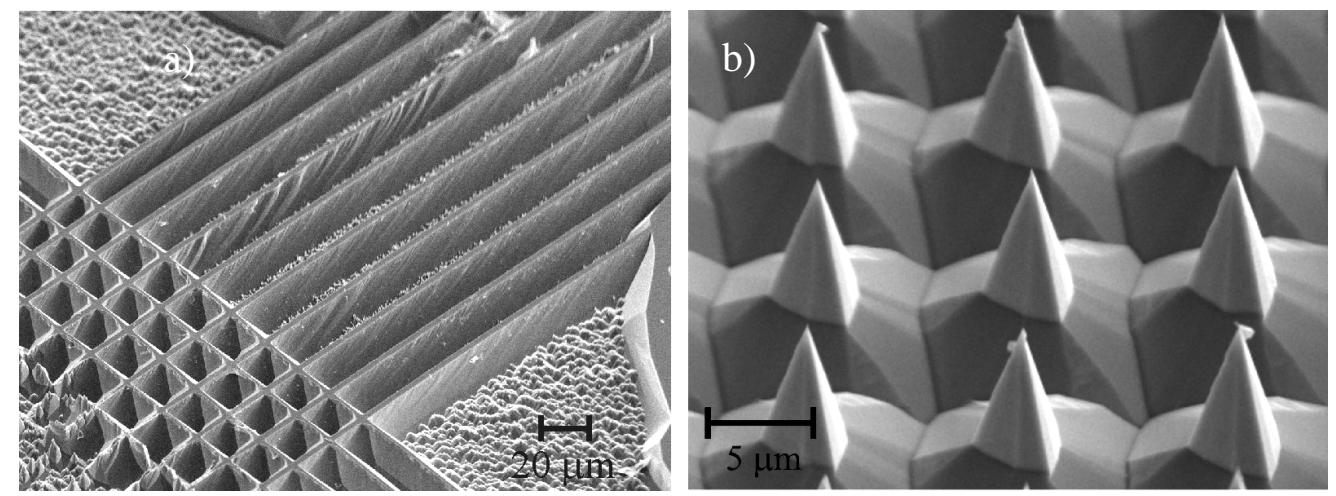

Figure 6. High aspect ratio structures produced by prolonged etching of pole-inhibited domains; a) cross hatch pattern produced by crossed overlapped scanned UV laser linear tracks and b) sharp tipped structures produced by static irradiation.
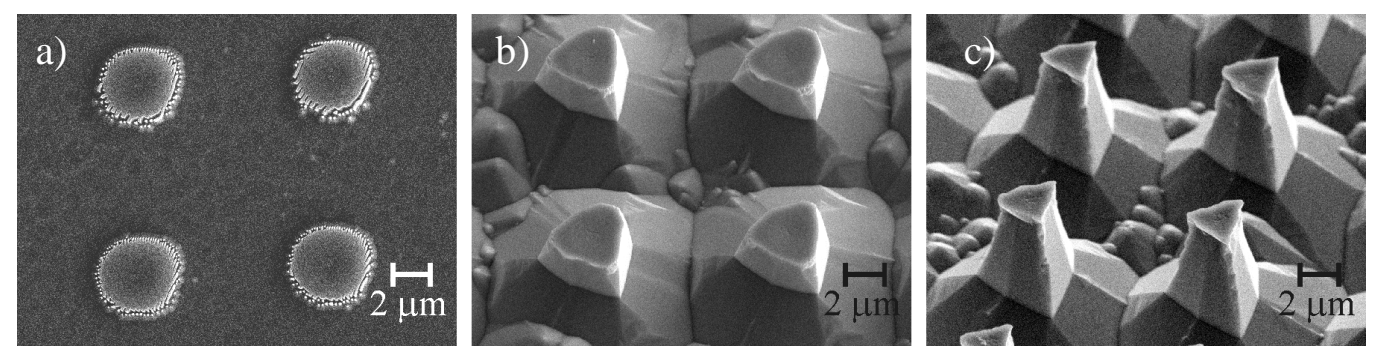

Figure 7. SEM images of isolated pole inhibited domains etched in HF acid for a) 15 minutes, b) $3.5 \mathrm{hrs}$, and c) $10 \mathrm{hrs}$. In b, and c, the sample was tilted by $30^{\circ}$ and $45^{\circ}$ respectively to make the undercut in the etched pillar visible

High aspect ratio structures can be produced in this manner examples of which are shown in figure 6. Figure 6a shows the surface topography of the cross hatched structure (part of which is shown in figure 4a) after etching in HF for $36 \mathrm{hrs}$. The height of the ridges is identical to the height of the bulk domain which is visible in the bottom left side of the SEM image. The sharp pyramidal features which are shown in figure $6 \mathrm{~b}$ correspond to long-etched isolated circular domains. 
The finite depth of the pole inhibited domains also produces undercuts after prolonged etching which is another fascinating property of these finite depth domains. The result of progressive etching of a single round pole inhibit domain is shown in the sequence of SEM images which is shown in figure 7. The undercut that develops in figure $7 \mathrm{~b}$ and $7 \mathrm{c}$ is associated is a result of sideways anisotropic etching and reflect the three fold symmetry directions on the $x-y$ plane of the crystal [34]. This is also the reason for the transformation of the originally round shape of the domain into a triangle as seen in the SEM image sequence of figure 7. Further etching results in complete elimination of the capping etch-stop layer and the pillars degenerate to sharp-tipped pyramids like the ones shown in figure $6 \mathrm{~b}$.

\section{Conclusions}

An outline of UV laser-induced and UV laser-assisted ferroelectric domain engineering methods applied to congruently melting LN crystals have been presented here. Irradiation of the polar surfaces with intense UV laser light which is strongly absorbed by the material was shown to directly induce or to inhibit local ferroelectric domain inversion depending on the polarity of the surface. It was concluded that it is the local heating of the crystal due to the strong absorption of the UV laser radiation and the corresponding large temperature gradients which are produced that are responsible for the observed ferroelectric domain behaviour. The actual physical processes behind the observed effects are not known with certainty due to the complexity of the physical problem. However, the role of lithium diffusion under the influence of the temperature gradients as well as transient electric fields which are formed due to changes in the spontaneous polarization during the heating cooling cycle is currently the subject of research regarding the physical origin of the experimental observations.

The ferroelectric domain distributions which are produced by the UV direct writing processes can overlap well with optical waveguide modes for nonlinear optical applications. Additionally, the potential of these methods for surface micro-structuring has been explored and it was shown that high aspect ratio surface micro-structures can be fabricated by differential etching utilizing shallow inverted domains as etch-stop layers. This is of particular interest especially when combined with the inherent ability of UV radiation to produce optical waveguides in $\mathrm{LN}$ for the fabrication of ridge waveguide photonic circuits.

\section{Acknowledgements}

Financial support from the European Commission in the framework of the STREP project 3D-DEMO is gratefully acknowledged.

\section{References}

1 R. S. Weis and T. K. Gaylord, Appl. Phys. A, 37, pp.191-203 (1985).

2 E. L. Wooten, K.M. Kissa, A. Yi-Yan, E. J. Murphy, D.A. Lafaw, P. F. Hallemeier, D. Maack, D.V. Attanasio, D. J. Fritz, G. J. McBrien, and D. E. Bossi, IEEE J. of Select. Topics in Quant. Elec. 6(1), pp.69-82 (2000).

3 M. Yamada, N. Nada, M. Saitoh, and K. Watanabe, Appl. Phys. Lett., 62(5), pp.435-436 (1993)

4 A. A. Oliver, Ed., Acoustic Surface Waves, Topics in Applied Physics, edited by A. A. Oliver (Springer, NewYork, 1978)

5 W. K. Burns, P. H. Klein, E. J. West, and L. E. Plew, J. Appl. Phys. 50, pp.6175-6182 (1979).

6 G. J. Griffiths and R. J. Esdaile, IEEE J. Quantum Electron. QE-20, 149 (1984).

7 J. L. Jackel, C. R. Rice, and J. J. Veselka, Appl. Phys. Lett. 41, pp.607-609 (1982).

8 Y. N. Korkishko and V. A. Fedorov, J. Appl. Phys. 82, pp.1010-1017 (1997) 
9 H. Hu, F. Lu, F. Chen, B-R. Shi, K-M. Wang, and D-Y. Shen, J. Appl. Phys. 89, pp.5224$5226(2001)$

10 R. Gattass and E. Mazur, Nature Photonics 2, pp. 219-225 (2008)

11 J. A. Armstrong, N. Bloembergen, J. Ducuing, and P. S. Pershan, Phys. Rev., 127, pp.1918-1939 (1962).

12 D. Janner, D. Tulli, M. García-Granda, M. Belmonte, and V. Pruneri, Laser \& Photon. Rev. 3, pp.301-313 (2009)

13 H. Gnewuch, N. K. Zayer, C. N. Pannell, G. W. Ross, and P. G. R. Smith Opt. Lett. 25, pp.305-307 (2000)

14 D. Yudistira, S. Benchabane, D. Janner, and V. Pruneri, Appl. Phys. Lett. 95, 052901 (2009)

15 I. E. Barry, G. W. Ross, P. G. R. Smith, R. W. Eason, and G. Cook, Mat. Lett. 37, pp.246254 (1998)

16 C. L. Sones, S. Mailis, V. Apostolopoulos, I. E. Barry, C. B. E. Gawith, P. G. R. Smith, and R. W. Eason, J. Micromech. Microeng. 12, pp.53-57 (2002)

17 . Müller, E. Soergel, and K. Buse, Appl. Phys. Lett., 83(9), pp.1824-1826 (2003).

18 C. L. Sones, M. C. Wengler, C. E. Valdivia, S. Mailis, R. W. Eason, and K. Buse, Appl. Phys. Lett., 86, 212901 (2005).

19 V. Dierolf and C. Sandmann, Appl. Phys. Lett., 84(20), pp3987-3989 (2004).

20 Y. J. Ying, C. E. Valdivia, C. L. Sones, R. W. Eason, and S. Mailis, Opt. Exp. 17, 18681 (2009)

21 A. C. Muir, G. J. Daniell, C. P. Please, I. T. Wellington, S. Mailis, and R. W. Eason, Appl. Phys. A: Mater. Sci. Process. 83, pp.389-396 (2006)

22 S. Mailis, C. Riziotis, I. T. Wellington, P. G. R. Smith, C. B. E. Gawith, and R. W. Eason, Opt. Lett. 28, pp.1433-1435 (2003)

23 C. L. Sones, S. Mailis, W. S. Brocklesby, R. W. Eason, and J. R. Owen, J. Mat. Chem., 12, pp.295-298 (2002)

24 S. Mailis, C. Riziotis, P. G. R. Smith, J. G. Scott, and R. W. Eason, Appl. Surf. Sci., 206, pp.46-52 (2003)

25 P. T. Brown, S. Mailis, I. Zergioti, and R. W. Eason, Opt. Mat. Vol.20(2) pp.125-134 (2002)

26 A. C. Muir, C. L. Sones, S. Mailis, R. W. Eason, E. Soergel, T. Jungk, and A. Hoffmann, Opt. Exp., 16, pp.2336-2350 (2008)

27 S. Mailis, P. T. Brown, C. L. Sones, I. Zergioti, and R. W. Eason, Appl. Phys. A, 74, pp.135-137 (2002)

28 R. G. Batchko, V. Y. Shur, M. M. Fejer, and R. L. Byer, Appl. Phys. Lett., 75, pp.16731675 (1999)

29 C. L. Sones, A. C. Muir, Y. J. Ying, S. Mailis, R. W. Eason, T. Jungk, A. Hoffmann, and E. Soergel, Appl. Phys. Lett., 92, 072905 (2008)

30 F. Johann, Y. J. Ying, T. Jungk, A. Hoffman, C. L. Sones, R. W. Eason, S. Mailis, and E. Soergel, Appl. Phys. Lett., 94, 172904 (2009)

31 D. Redfield, and W. J. Burke, J. Appl. Phys. 45, pp.4566-4571 (1974) 
32 A. M. Mamedov, Opt. Spectrosc. 56, pp.645- (1984)

33 S. Mailis, C. L. Sones, and R. W. Eason, Micro-/Nanoengineering and Characterization of Ferroelectric Crystals for Photonic Applications Series: Springer Series in Materials Science, Vol. 91 ed. P. Ferraro, S. Grilli, P. De Natale, 2008

34 T. J. Sono, J. G. Scott, C. L. Sones, C. E. Valdivia, S. Mailis, R. W. Eason, J. G. Frey, and L. Danos, Phys. Rev. B, 74, 205424 (2006) 